\title{
Survival benefit of immune checkpoint inhibitor monotherapy in patients with non-small cell lung cancer recurrence after completely pulmonary resection
}

\author{
Hiroaki Kuroda ${ }^{1}$, Yusuke Takahashi ${ }^{1,2}$, Suguru Shirai ${ }^{1}$, Hirotomo Takahara ${ }^{1}$, Takeo Nakada ${ }^{1}$, Noriaki Sakakura ${ }^{1}$, \\ Hirokazu Matsushita ${ }^{2}$ \\ ${ }^{1}$ Department of Thoracic Surgery, Aichi Cancer Center Hospital, Nagoya, Japan; ${ }^{2}$ Division of Translational Oncoimmunology, Aichi Cancer \\ Research Institute, Nagoya, Japan \\ Contributions: (I) Conception and design: H Kuroda, S Shirai; (II) Administrative support: Y Takahashi; (III) Provision of study materials or patients: \\ T Nakada, H Takahara; (IV) Collection and assembly of data: H Kuroda, N Sakakura; (V) Data analysis and interpretation: H Kuroda, H Matsushita; \\ (VI) Manuscript writing: All authors; (VII) Final approval of manuscript: All authors. \\ Correspondence to: Hiroaki Kuroda. Department of Thoracic Surgery, Aichi Cancer Center Hospital, 1-1, Kanokoden, Chikusa-ku, Nagoya 464-8681, \\ Japan. Email: h-kuroda@aichi-cc.jp.
}

Background: Selected patients in non-small cell lung cancer (NSCLC) responded to the treatment of immune checkpoint inhibitors (ICIs) have the survival benefit for advanced stages or metastatic status.

Methods: We investigated whether a response to ICI monotherapy since 2016 influences the survival of NSCLC patients with recurrence after completely pulmonary resection between 2009 and 2017. Disease control rate (DCR) was calculated as complete plus partial response plus stable disease during more than 6 months.

Results: Thirty-five patients (mean age 67 years, range $46-79$ years, 60\% male) were included in the study. The most frequent histology and pathological stage were adenocarcinoma (60\%) and IIB (45.7\%), respectively. ICI was used at a median of second-line treatment. The DCR and median progressionfree survival were $42.8 \%$ and 2.5 (95\% CI: 1.6-3.4) months, respectively. The therapeutic outcome from recurrence was $47.5 \%$. Multivariate analysis revealed a significant impact of DCR on favorable therapeutic outcome $(\mathrm{P}=0.04)$. A serial increase (pre- to post-surgery to ICI initiation) of C-reactive protein $(\mathrm{CRP})$ and prognostic nutritional index (PNI) was associated with treatment response (both $\mathrm{P}=0.01$ ).

Conclusions: These results suggest that a response to ICI monotherapy significantly contributes to a survival benefit regardless of therapeutic lines in NSCLC patients with recurrence after completely pulmonary resection, and the therapeutic response is strongly associated with a serial increase in CRP or decrease in prognostic nutritional index.

Keywords: Immune checkpoint inhibitor (ICI); non-small cell lung cancer (NSCLC); C-reactive protein (CRP); prognostic nutritional index

Submitted Mar 27, 2021. Accepted for publication Jun 11, 2021.

doi: 10.21037/atm-21-1492

View this article at: https://dx.doi.org/10.21037/atm-21-1492

\section{Introduction}

Pulmonary resection is of strategic importance for the treatment of clinical stage IA-IIIA non-small cell lung cancer (NSCLC). Although completely resection is crucial for improving the prognosis of patients with NSCLC, a completely cure cannot be attained even with nearly perfect resection or in cases of early-staged NSCLC $(1,2)$. A recent trial of MAGE-A3 immunotherapy as an adjuvant NSCLC treatment revealed that $36.2 \%$ of surgically resected stage IB-IIIA NSCLCs were cancer-specific events, including 
$35.8 \%$ cases of loco-regional relapse, $58.8 \%$ cases of distant metastases, and $5.4 \%$ cases of a second primary lung cancer, during the median 40 months of follow-up (3). In addition, the recurrence rate was the highest in the first 18 months after surgery, with a peak between 6 and 12 months, and decreased thereafter (1). The consequent treatment following diagnosis of a recurrence is based on the therapeutic guideline for advanced NSCLC (4).

Since the early 2000s, new treatment strategies for NSCLC have emerged, including molecular target drugs or immune checkpoint inhibitors (ICIs), based on sufficient evidence of their effectiveness compared with conventional cytotoxic anticancer agents. Over the last decade, the treatment of advanced NSCLC has ameliorated dramatically in selected patients, especially with the introduction of ICIs. ICIs can restore the immune system through the disruption of intrinsic ligand-receptor interactions. However, responses to ICI monotherapy are clinically scattered, and the prognostic factors are still not well defined. We previously reported that ICI monotherapy was significantly less efficacious in patients with anaplastic lymphoma kinase $(A L K)$ gene rearrangement than in patients with epidermal growth factor receptor (EGFR) mutations, and that programmed death-ligand 1 (PD-L1) expression was not a critical biomarker to predict the therapeutic response for patients with one of these mutations (5). However, elucidation of the detailed molecular mechanisms underlying treatment resistance and the development of drug or combination therapy to overcome resistance is challenging.

In particular, a definitive biomarker for prediction of the therapeutic efficacy of ICI is still required. To date, tumor signatures such as PD-L1 expression and the tumor mutational burden have been most widely used as predictive biomarkers (6). Systemic inflammation is strongly associated with a poor prognosis of patients with advanced NSCLC; however, there is insufficient evidence about the responses to ICI therapies under an inflammatory status. We previously reported that elevated C-reactive protein (CRP) levels ( $>1 \mathrm{mg} / \mathrm{dL})$ and a lactate dehydrogenase (LDH) level greater than the normal upper range were significantly associated with the response duration and survival in patients treated with nivolumab (7). Perioperatively, the blood works were performed two days before surgery, and 1 month after surgery. Recently, numerous studies have demonstrated that a high neutrophil-to-lymphocyte ratio (NLR) is a useful marker of host inflammation and is also associated with poorer survival in patients with various cancers, including NSCLC (8). In addition, we previously reported that prognostic nutritional index (PNI) was significant prognostic factor in stage I NSCLCs after completely pulmonary resection (9).

The aim of this retrospective study was to investigate whether the response to ICI monotherapy had an influence on the survival of NSCLC patients with recurrence after completely pulmonary resection. We also evaluated whether the inflammatory status in the peripheral blood was associated with a response [disease control rate (DCR)] to ICI monotherapy. As a radiological biomarker, ${ }^{18} \mathrm{~F}$-flurodeoxyglucose positron emission tomography/ computed tomography $\left({ }^{18} \mathrm{~F}-\mathrm{FDG}\right.$ PET/CT) reflects the interaction between the metabolic tumor burden and immune pathways (10). Therefore, we also evaluated the relationship between the parameters of ${ }^{18} \mathrm{~F}-\mathrm{FDG}$ PET/ $\mathrm{CT}$, such as the maximum standard uptake value (SUVmax) and tumor lesion glycolysis (TLG), and the response to ICI monotherapy after recurrence. We present the following article in accordance with the STROBE reporting checklist (available at https://dx.doi.org/10.21037/atm-21-1492).

\section{Methods}

\section{Patients}

We retrospectively analyzed the clinical data of 51 patients diagnosed with recurrence after completely pulmonary resection for NSCLC who received ICI monotherapy since January 2016 during the therapeutic course, including nivolumab, pembrolizumab, atezolizumab, and ipilimumab, at Aichi Cancer Center Hospital. One hundred eight hundred twenty-five patients underwent pulmonary completely resection between December 2009 and October 2017, and 381 patients (20.9\%) were diagnosed as recurrence. Pre-operative and post-operative laboratory measurements at 1 month, and data on pretreatment with ICI and PET/CT results before ICI initiation after recurrence were available for $35(68.6 \%)$ patients. The database was closed on October 1, 2020; at that time, $62.9 \%$ $(22 / 35)$ of the patients included in the study had died. This study was conducted in accordance with the Declaration of Helsinki (as revised in 2013). This study was approved by the Institutional Review Board of Aichi Cancer Center Hospital (ACC-2019-1-003). The requirement of informed consent was waived because of the retrospective nature of this study. CRP, LDH, and NLR, and the PNI (10x serum albumin+lymphocyte counts $\times 0.05$ ) were measured before 
surgery, at 1 month after the operation, and before the initiation of ICI. Data on patient characteristics, genetic status $[E G F R,-\mathrm{Ki}$-ras2 Kirsten rat sarcoma viral oncogene homolog (KRAS), $A L K$ ], tumor proportion score (TPS), ICI response, PFS, and therapeutic outcomes were obtained from clinical records. LDH and CRP levels were measured just before the initiation of treatment with ICI. We used the following cut-off levels for comparison: LDH, $<245$ vs. $\geq 245 \mathrm{IU} / \mathrm{L}$; CRP, $<1.0$ vs. $\geq 1.0 \mathrm{mg} / \mathrm{dL}$; PNI, $<50$ vs. $\geq 50 \mathrm{mg} / \mathrm{dL}$; and NLR, $<3 v s . \geq 3$, as previously reported (5).

The TPS was evaluated using surgically resected specimens with an automated immunohistochemistry assay as reported previously (5). EGFR mutations (exons 18-21) were identified using polymerase chain reaction. $A L K$ fusions were examined by reverse transcription-polymerase chain reaction or fluorescence in situ hybridization (Vysis ALK Break Apart FISH Probe Kit; Vysis, Inc., Downers Grove, IL, USA) after immunohistochemistry screening, as previously reported (5).

In the follow-up duration, chest to upper abdominal computed tomography was routinely performed on a semi-annual basis. Brain magnetic resonance imaging and positron emission tomography were added 1, 2 and 5 years after surgery.

\section{Evaluation of the efficacy $f$ ICI monotherapy}

The context of ICIs including the registration of clinical trials at the initial administration after the diagnosis of recurrence is decided by our institutional cancer board comprising thoracic surgeons, oncologists, and radiologists. The patients were treated with an ICI until they showed disease progression or experienced unacceptable adverse events. In general, the patients underwent radiographic imaging every 2 months during treatment and were evaluated for tumor response according to the Response Evaluation Criteria in Solid Tumors, version 1.1. The DCR was calculated as complete plus partial response plus stable disease during more than 6 months. We used DCR as a response to ICI therapy in this study.

\section{Statistical analysis}

All data were analyzed using Statistical Package for the Social Sciences (SPSS) software (version 25.0, SPSS Institute Inc., Chicago, IL, USA). Categorical variables were compared using the chi-square test. Differences among two and three groups were evaluated using the Mann-Whitney and the Kruskal-Wallis test. Survival rates were analyzed using the Kaplan-Meier method and compared between patient groups using the log-rank test. Cox proportional hazards regression analysis was performed to investigate the factors related to survival after recurrence. Covariates with a $\mathrm{P}$ value of $<0.10$ in the univariate analysis were included in the multivariate model. Statistical significance was set at $\mathrm{P}<0.05$.

\section{Results}

\section{Patient characteristics and treatment}

Fifty-One patients diagnosed with recurrence after completely pulmonary resection for NSCLC who received ICI monotherapy during the therapeutic course. The CONSORT flowchart for patient selection and inclusion is shown in Figure 1. Thirty-five patients (68.6\%) were candidates in this retrospective and single-institutional study. The clinical and demographic characteristics of the patients are summarized in Table 1 . The median age was 67 years, and 30 of the 35 patients $(85.7 \%)$ underwent lobectomy, with a median duration of 15.0 months between pulmonary resection and recurrence. Sixteen patients (45.7\%) experienced distant metastases. The most frequent histology and pathological stage was adenocarcinoma $(60.0 \%)$ and p-IIB (45.7\%), respectively, and the majority of patients were ex-smokers. The median time from surgery to recurrence was 15.0 months (interquartile range; 8.9 to 21.9). The recurrence sites were locoregional in $19(54.3 \%)$ and distant in 16 (45.7\%). The Eastern Cooperative Oncology Group Performance Status score before starting with ICIs was 0 for 10 patients (28.6\%), 1 for 22 patients (62.9\%), and 2 for 3 patients (8.5\%). ICI was used as the second-line or higher treatment for the majority of patients. Most of the patients were treated with nivolumab (65.7\%), followed by pembrolizumab (25.7\%). EGFR mutations were the most common, followed by KRAS mutations, and one patient had $A L K$ rearrangement. The PD-L1 expression status could be evaluated in $28(80 \%)$ patients, seven of whom showed high PD-L1 expression (>50\%), 10 had low PD-L1 expression (1-49\%), and 11 were PD-L1-negative.

\section{Clinical outcomes following ICI monotherapy or according to mutational status}

According to the Immune Response Evaluation Criteria in Solid Tumors, 9 patients $(25.7 \%)$ showed a partial 


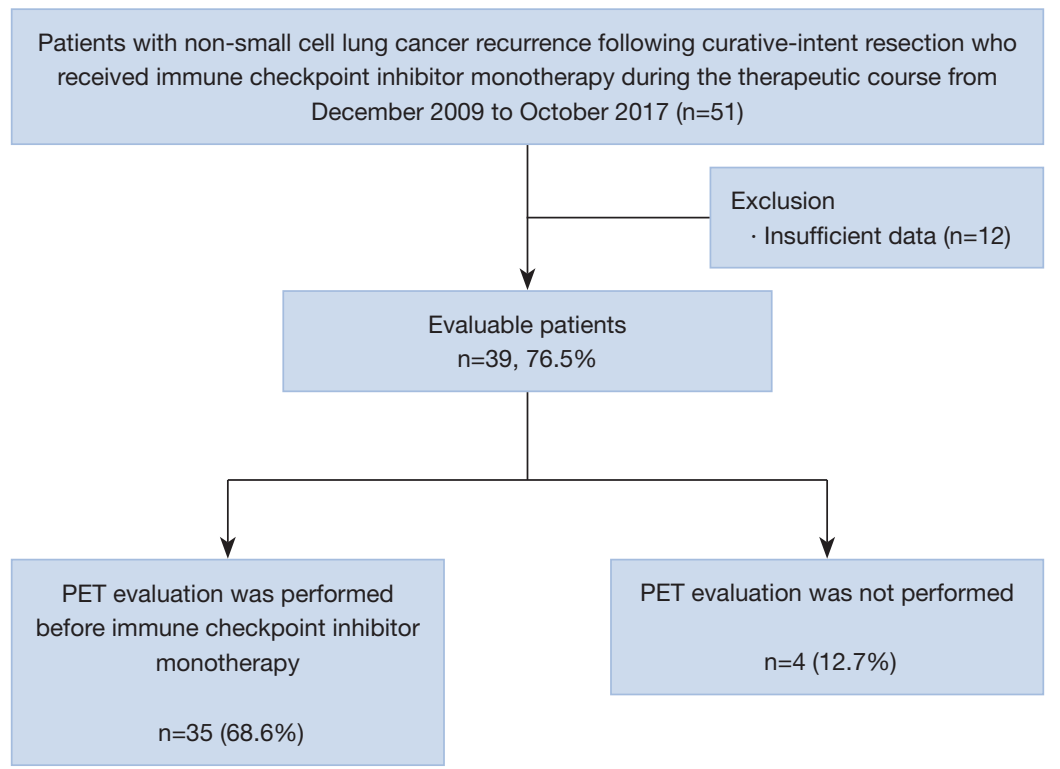

Figure 1 Patient flowchart.

response, $6(17.1 \%)$ showed a stable disease, and 20 (57.2\%) had progressive disease. With respect to efficacy measurements, DCR and median progression-free survival (PFS) were $42.8 \%$ and 2.5 (95\% CI: 1.6-3.4) months, respectively (Figure $2 A$ ). The proportion of response for ICI was increased according to the TPS score $(\mathrm{P}<0.01)$ (Figure S1). In addition, the 5 -year therapeutic outcome from recurrence was equivalent between $1^{\text {st }}$ and $2^{\text {nd }}$ lines and more than $2^{\text {nd }}$ line $[46.6 \%(\mathrm{n}=19)$ vs. $49.2 \%(\mathrm{~N}=16)$; $(\mathrm{P}=0.08)]$.

\section{Clinical outcomes according to the mutational status or preradiotherapy before ICIs}

While there is no significant difference in 5-year therapeutic outcomes from recurrence with or without ALK or EGFR [75.0\% ( $\mathrm{n}=8)$ vs. 37.1\% ( $\mathrm{N}=27) ;(\mathrm{P}=0.08)]$ (Figure $\mathrm{S} 2 \mathrm{~A})$. Eight patients $(8 / 35,22.9 \%)$ received local radiotherapy before ICI. There was no significant difference in the therapeutic outcomes after recurrence with or without radiotherapy (40.0\% vs. $48.2 \%, \mathrm{P}=0.98$ ) (Figure $\mathrm{S} 2 \mathrm{~B}$ ).

\section{Impact of response to ICI monotherapy on survival after recurrence}

We next investigated whether the response (DCR) to ICI monotherapy influences the clinical outcomes after recurrence using univariate and multivariate regression analyses. The median follow-up duration was 58.1 months [interquartile range (IQR) 15.5-105.8 months] and 39.1 (IQR 16.0-56.8 months) after pulmonary resection and from diagnosis of recurrence, respectively. The 2-year and 5-year therapeutic outcome rates from recurrence after pulmonary resection and from diagnosis of recurrence were $68.3 \%$ and $47.5 \%$, respectively (Figure $2 B$ ). Univariate and multivariate analyses revealed that only the response (DCR) to ICI monotherapy had a statistically significant association with a favorable PFS ( $\mathrm{P}=0.03$ and $\mathrm{P}=0.04$, respectively) (Table 2 ). The therapeutic outcomes after recurrence in DCR was better than those in no DCR (62.9\% vs. $35.0 \%, \mathrm{P}=0.02)$ (Figure S2C).

\section{Impact of laboratory parameters on the response (DCR) to ICI monotherapy}

We next evaluated the influence of laboratory parameters on the response to ICI monotherapy, including CRP, LDH, PNI, and NLR. As shown in Table 3, none of these parameters significantly changed from the pre- to postpulmonary resection stage. However, an increase in the CRP level to $>1.0 \mathrm{mg} / \mathrm{dL}$ and a decrease in the PNI to $<50$ from post-surgical resection to the initiation of ICI were significantly associated with a response to ICI monotherapy. In addition, serial increases (pre- to post- 
Table 1 Clinicopathological characteristics of the included patients $(\mathrm{N}=35)$

\begin{tabular}{|c|c|}
\hline Characteristics & Value \\
\hline Sex, male (\%) & $21(60.0)$ \\
\hline Smoking history, $\mathrm{n}(\%)$, pack-years (mean $\pm \mathrm{SD}$, range) & 28 (80.0), 51.9 $25.5(42.0-61.8)$ \\
\hline \multicolumn{2}{|l|}{ Histology } \\
\hline \multicolumn{2}{|l|}{ Type of procedures } \\
\hline Pneumonectomy/lobectomy/sublobar (n) & $2 / 30 / 3$ \\
\hline Adjuvant chemotherapy, n (\%) & $19(54.3)$ \\
\hline \multicolumn{2}{|l|}{ Pathological stage (n) } \\
\hline IIIA/IIIB & $8 / 1$ \\
\hline \multicolumn{2}{|l|}{ Genomic mutations, n (\%) } \\
\hline EGFR & $7(20.0)$ \\
\hline KRAS & $6(17.1)$ \\
\hline$A L K$ & $1(2.9)$ \\
\hline No mutations & $21(80.0)$ \\
\hline \multicolumn{2}{|l|}{ Treatment, n (\%) } \\
\hline Nivolumab (anti-PD-1) & $23(65.7)$ \\
\hline
\end{tabular}

$\mathrm{SD}$, standard deviation; EGFR, epidermal growth factor receptor; $A L K$, anaplastic lymphoma kinase; KRAS, v-Ki-ras2 Kirsten rat sarcoma viral oncogene homolog; PDL1, program death-ligand 1; CTLA, cytotoxic T-lymphocyte antigen 4.

surgery to ICI initiation) of CRP and serial decreases of NLR were significantly associated with a treatment response.

\section{Influence of PET parameters on the response to ICI monotherapy and survival after recurrence}

Representative results are shown in Figure 3A. The median SUVmax and TLG were higher among ICI responders than non-responders (Figure 3B,3C), but the differences were not statistically significant [SUVmax median (IQR): 16.4 (7.9-21.9) vs. 10.8 (5.7-16.5), $\mathrm{P}=0.12$; TLG median (IQR):
26.7 (9.6-86.3) vs. $25.3(7.3-59.5) ; \mathrm{P}=0.53]$.

\section{Discussion}

The present study was designed to determine the impact of the response to ICI monotherapy on the survival of NSCLC patients with recurrence after completely pulmonary resection. In addition, we conducted a retrospective analysis of laboratory or radiological parameters prior to ICI monotherapy that may influence the therapeutic response in the same period. The DCR and median PFS were $42.8 \%$ and 2.5 months, respectively. The 

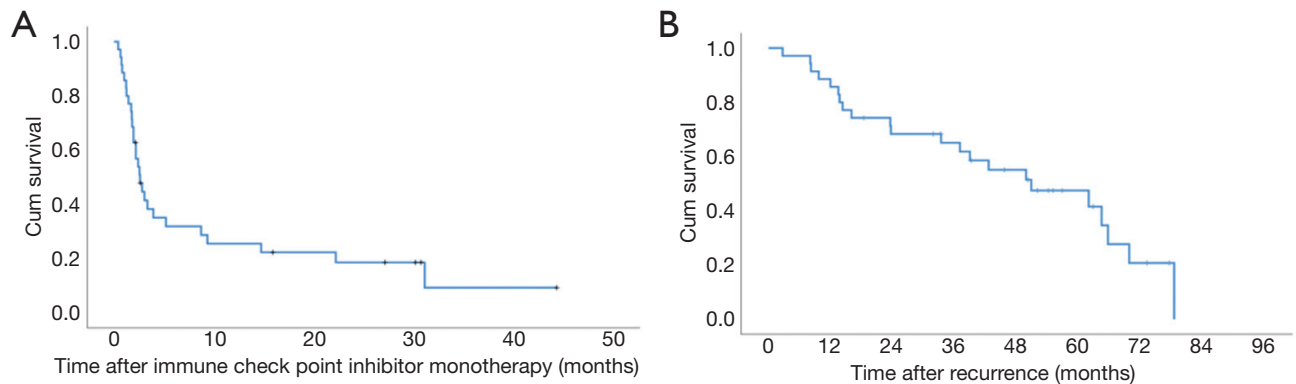

Figure 2 Clinical outcomes in patients with recurrence after completely pulmonary resection. (A) Progression-free survival after immune checkpoint inhibitor monotherapy. And (B) overall survival after diagnosis of recurrence.

Table 2 Univariate and multivariate analyses according to the Cox proportional hazard model for the impact of ICI response on progression-free survival

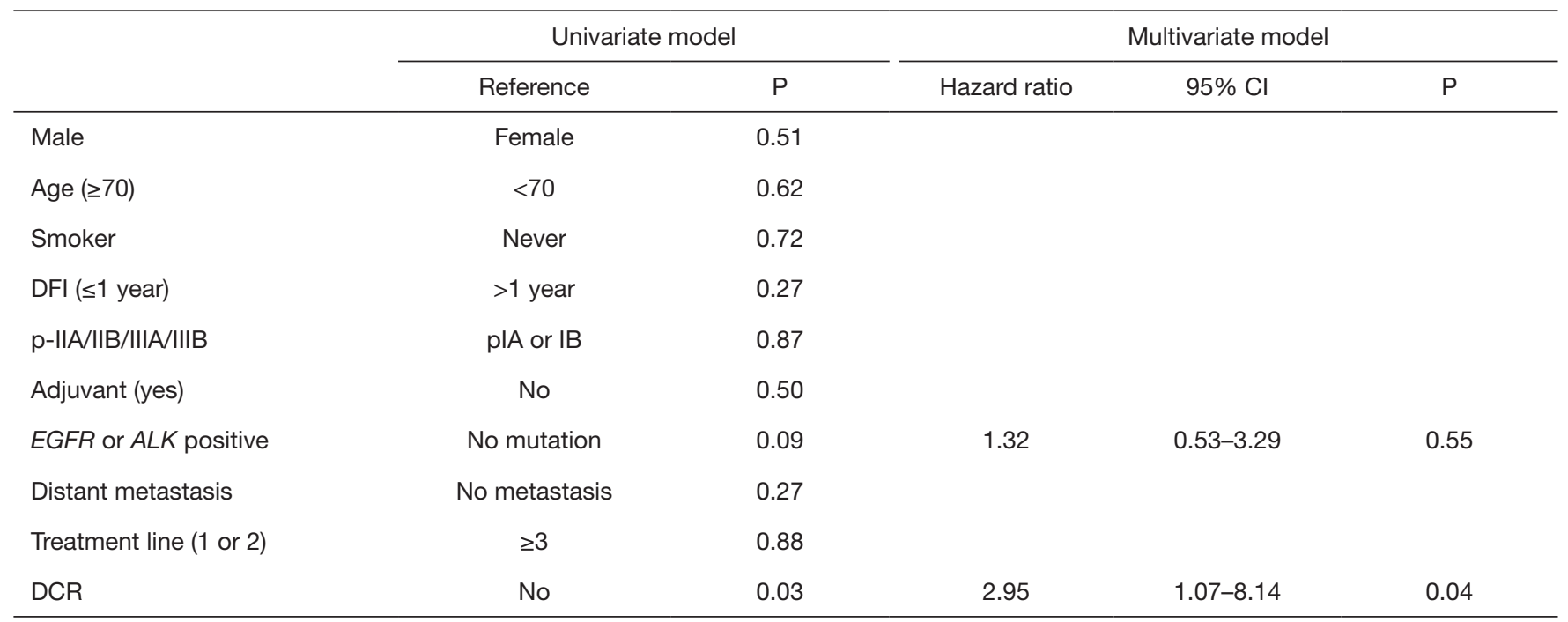

$\mathrm{DFI}$, disease-free intervals; $A L K$, anaplastic lymphoma kinase; $E G F R$, epidermal growth factor receptor; DCR, disease control rate; Cl, confidence intervals.

PFS found in this study is compatible with that reported in previously published clinical trials of ICI monotherapy (11-13). Multivariate analysis including variables that were significantly associated with survival in univariate analysis showed that only the response to ICI monotherapy was independently statistically associated with a survival benefit $(\mathrm{P}=0.04)$. Although no significant difference was found in factors closely associated with the response to ICI monotherapy, laboratory parameters changed from pre- to post-pulmonary resection and from post-surgical resection to the initiation of ICI. There was no significant effect of PET parameters on the response to ICI monotherapy. In addition, there is no radiological modality or tracer being widely in the clinical practice than can determine the immune tumor microenvironment. In other words, the use of ${ }^{18} \mathrm{~F}$-FDG PET for effect measurement is still controversial. However, the results of this study could not identify the factors such as CRP or PNI that are most closely associated with the response to ICI monotherapy in patients that experience a recurrence following pulmonary resection for NSCLC, necessitating further investigation and accumulation of clinical data to resolve this question. Nevertheless, favorable survival was strongly associated with a response to ICI monotherapy after recurrence.

This study suggests that the response to ICI monotherapy plays a key role in prolonging the lifetime from the diagnosis of recurrence, regardless of the therapeutic line. In the KEYNOTE-042 trial, pembrolizumab monotherapy 
Table 3 Relationship of laboratory parameters with the disease control rate to ICI monotherapy

\begin{tabular}{|c|c|c|c|c|}
\hline Variables & Value & $\mathrm{HR}$ & $95 \% \mathrm{Cl}$ & $\mathrm{P}$ \\
\hline \multirow[t]{3}{*}{ CRP } & Increase & 1.65 & $0.26-10.3$ & 0.61 \\
\hline & Increase +1.0 mg/dL & 1.63 & $0.41-6.39$ & 0.49 \\
\hline & Increase $+2.0 \mathrm{mg} / \mathrm{dL}$ & 2.06 & $0.39-11.0$ & 0.40 \\
\hline PNI & Decrease $+<50$ & 2.75 & $0.65-11.6$ & 0.17 \\
\hline NLR & Increase $+>3$ & 1.56 & $0.38-2.63$ & 0.64 \\
\hline \multicolumn{5}{|c|}{ Post-operation to initiation of $\mathrm{ICI}$} \\
\hline CRP & Increase & 11.0 & $2.3-53.6$ & $<0.01^{*}$ \\
\hline LDH & Increase + upper limit of normal & 1.09 & $0.24-5.03$ & 0.91 \\
\hline $\mathrm{PNI}$ & Decrease $+<50$ & 0.12 & $0.26-0.56$ & $<0.01^{*}$ \\
\hline NLR & Increase $+>3$ & 0.33 & $0.08-1.35$ & 0.12 \\
\hline \multicolumn{5}{|c|}{ Pre- to post-operation and post-operation to initiation of $\mathrm{ICl}$} \\
\hline CRP & Both increase & 0.10 & $0.02-0.58$ & $0.01^{*}$ \\
\hline LDH & Both increase & 0.87 & $0.13-6.00$ & 0.89 \\
\hline $\mathrm{PNI}$ & Both decrease & 0.10 & $0.02-0.58$ & $0.01^{*}$ \\
\hline NLR & Both increase & 0.69 & $0.14-3.35$ & 0.64 \\
\hline
\end{tabular}

${ }^{*} \mathrm{P}<0.05$. CRP, C-reactive protein; LDH, lactate dehydrogenase; PNI, prognostic nutritional index; NLR, neutrophil-to-lymphocyte ratio; ICI, immune checkpoint inhibitor; $\mathrm{HR}$, hazard ratio; $\mathrm{Cl}$, confidence intervals.

was associated with significantly improved PFS with a hazard ratio of 0.50 (95\% CI: $0.37-0.68, \mathrm{P}<0.001)$ compared with platinum-based chemotherapy in patients with advanced NSCLC with a TPS of $\geq 50 \%$ and without EGFR mutation or $A L K$ rearrangement (14). This trial also showed that a TPS $\geq 50 \%$ contributed to a favorable prognostic profile with first-line ICI monotherapy (14). Several recent clinical trials have postulated that the synergistic efficacy of a combination of platinum-doublet chemotherapy could provide a survival benefit and high treatment response rate $(15,16)$. However, there can also be indications to ease concern over the loss of regional lymph nodes rather than the removal of metastases by surgical lymph node dissection, which might accelerate the loss of offensive troops with immunological sensitization closest to cancers, such as cytotoxic T lymphocytes, dendritic cells, and $\gamma$-globulin. A study in a small Japanese cohort reported no significant difference in outcomes with or without surgery (17).

Lung adenocarcinoma in East Asia is regionally characterized by predominance of EGFR mutations with a high proportion of never-smokers (18). We previously reported distinct recurrence patterns after completely pulmonary resection according to oncogenic mutation status and mutational EGFR subtypes, in which EGFRpositive patients mostly experienced pleural recurrence, with a significantly higher incidence in patients with triplenegative mutations (19). In addition, the presence of EGFR mutation or $A L K$ rearrangement was a significant favorable prognostic factor for time to recurrence (18). Another cohort study showed that the most common recurrence site after completely resection of EGFR-mutant NSCLC was thoracic recurrence (20), which is compatible with the findings of our cohort. Although the effect of oncogenic driver mutation on the efficacy of ICI treatment remains unclear, several studies have demonstrated that oncogenic signals 

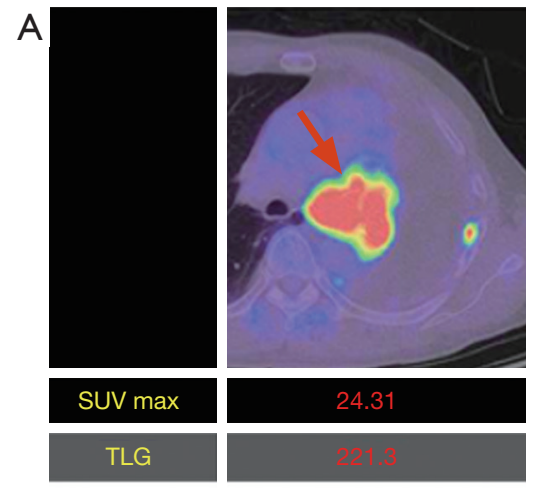

B

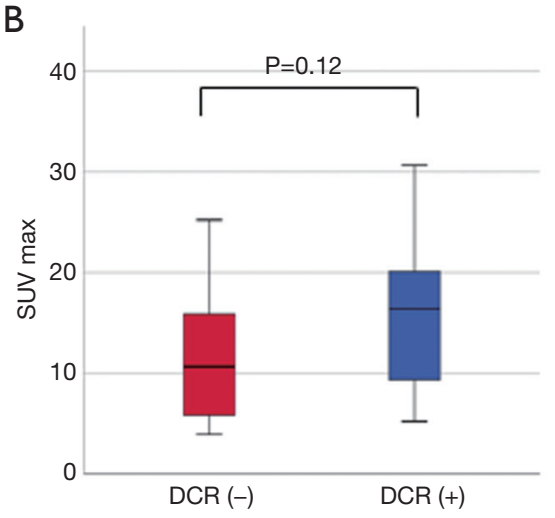

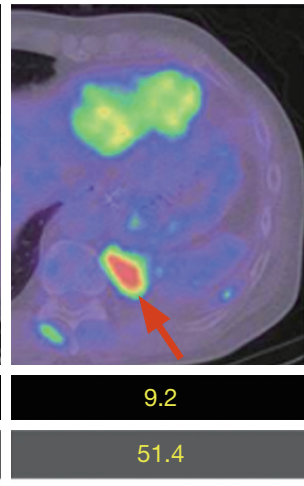

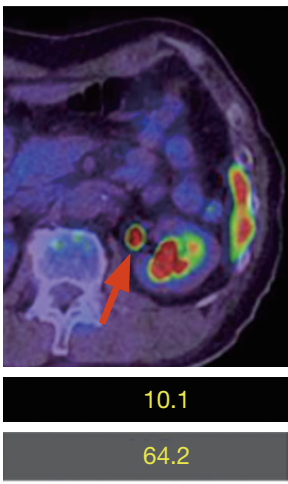

C

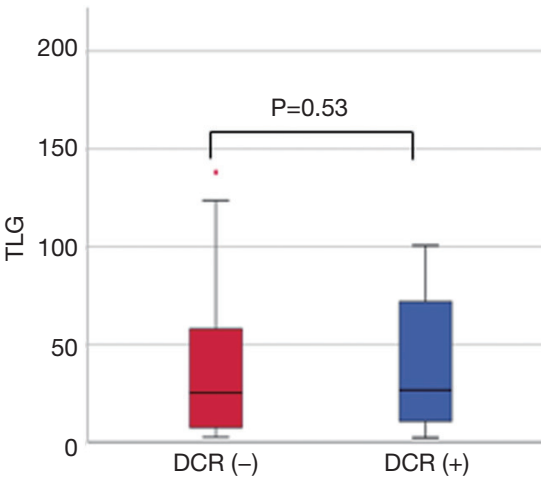

Figure 3 PET findings. (A) Representative maximum accumulation (red arrow). (Right) mediastinal lymph node; (central) abdominal lymph node; and (left) left adrenal. (B) Standard uptake value (SUVmax) based on disease control rate (DCR). And (C) tumor lesion glycolysis (TLG).

derived from mutations or loss of tumor suppressor genes upregulate the expression of immune checkpoint molecules in cancer cells as a mechanism of immune escape (5). In this study, the presence of EGFR mutation or $A L K$ rearrangement did not significantly influence the survival of patients with recurrence after completely pulmonary resection compared with ICI monotherapy. The results for the Japan subset in the KEYNOTE-024 trial indicated that first-line pembrolizumab improved the PFS and overall survival compared to chemotherapy, with manageable safety found among Japanese patients with metastatic NSCLC without $E G F R / A L K$ alterations and a TPS of $\geq 50 \%$ (21). Our previous study also revealed that PD-L1 expression may not be an effective predictor in patients with lung cancer who have $A L K$ or EGFR mutations. According to the National Comprehensive Cancer Network (NCCN) guideline, osimertinib or initial cytotoxic chemotherapy as subsequent therapy following tyrosine kinase inhibitor (TKI) failure is recommended depending on the presence of the T790M mutation.

In this study, we could not identify a novel biomarker for predicting ICI response from radiological findings. However, an increase of CRP above $1.0 \mathrm{mg} / \mathrm{dL}$ and a decrease in the PNI to $<50$ from the post-operative period to the initiation of ICI were closely associated with the response to ICI monotherapy $(\mathrm{P}=0.02$ and $\mathrm{P}<0.01$, respectively). In addition, the serial increases in CRP and a serial decrease of PNI from pre- to post-operation to the initiation of ICI were also closely associated with the treatment response $(\mathrm{P}=0.01$ and $\mathrm{P}=0.01$, respectively). Although the efficacy of ICI therapy has been discussed in terms of the cancer inflammation status, it remains poorly understood. We previously reported that elevated CRP (>1 mg/dL) and LDH levels above the normal upper range were significantly associated with the response duration and survival in patients treated with nivolumab (7). Numerous inflammatory-related parameters such as CRP, LDH, albumin level, NLR, and platelet-to-lymphocyte ratio, which are all associated with cancer prognosis, have also been reported as potential biomarkers for the ICI treatment response (22). However, few studies have focused on the association of serial changes in inflammationrelated parameters from the pre-operative stage to initial 
ICI treatment after recurrence with therapeutic efficacy. Although we did not find a significant association of the parameter change from pre- to post-operation with the ICI therapy response, significant differences were found in terms of the serial increase of CRP. Similar to our previous report, the change in CRP from the post-operative period to the initiation of ICI monotherapy and a CRP level $>1 \mathrm{mg} / \mathrm{dL}$ were significantly related to the therapeutic response (7).

Moreover, the change in PNI from the post-operative period to the initiation of ICI monotherapy and a serial decrease in PNI were closely associated with the response to treatment. We previously reported that the PNI is an independent prognostic factor for patient survival in NSCLC patients (9). Another study showed that sarcopenia or a poor performance status was strongly associated with PFS or the therapeutic effect of ICIs in NSCLC patients (23). Therefore, further research is needed to validate the biomarkers involved in the response to ICI therapies.

This study has several limitations. First, the retrospective nature with a small sample size conducted at a single institution introduce potential bias. Second, the greater prevalence of EGFR mutations in East Asian patients with NSCLC is well known, whereas the proportion of EGFR or $A L K$ mutations was only $22.9 \%(8 / 35)$ in our study, which is relatively lower than that of the general population. TKIs are firmly positioned for treatment following a diagnosis of recurrence given evidence of their high efficacy, and therefore it is difficult to verify the effect of ICIs with or without a treatment history of TKI. Accordingly, patients with $E G F R$ or $A L K$ mutations tend to receive ICIs as a late-line treatment because other candidates are preferred. Third, more than half of the patients $(51.4 \%)$ did not undergo PD-L1 testing $(\mathrm{n}=7)$ or were negative for PDL1 expression $(n=11)$. Therefore, we could not consider the effect of PD-L1 expression on the treatment response. Finally, we were only able to evaluate data for $68.6 \%$ (35/51) of all eligible patients during the study period. Most patients could not undergo PET evaluation before the initiation of ICI treatment. To overcome these limitations, prospective trials will be required to confirm the relative effect of the response to ICI or driver oncogenes on survival prolongation to gain better understanding of the efficacy of ICI treatment recurrence after completely pulmonary resection.

In conclusion, the response to ICI monotherapy significantly contributes to a survival benefit for patients with recurrent NSCLC following completely pulmonary resection, regardless of therapeutic lines. The response to ICI monotherapy was strongly associated with a serial increase of CRP or a serial decrease of the PNI before the operation, at 1 month after operation, and before the initiation of ICI. In addition, an increase in CRP to $>1 \mathrm{mg} / \mathrm{dL}$ and a decrease in the PNI to $<50$ before the initiation of ICI compared with the respective values 1 month after the operation were also closely associated with the response to treatment. Further investigation is needed to elucidate the efficacy or role of ICI treatment during the therapeutic course following post-surgical recurrence.

\section{Acknowledgments}

The abstract of this article was presented at ESTS 2019 in Dublin, Ireland, as a poster presentation.

Funding: None.

\section{Footnote}

Reporting Checklist: The authors have completed the STROBE reporting checklist. Available at https://dx.doi. org/10.21037/atm-21-1492

Data Sharing Statement: Available at https://dx.doi. org/10.21037/atm-21-1492

Peer Review File: Available at https://dx.doi.org/10.21037/ atm-21-1492

Conflicts of Interest: All authors have completed the ICMJE uniform disclosure form (available at https://dx.doi. org/10.21037/atm-21-1492). The authors have no conflict of interest to declare.

Ethical Statement: The authors are accountable for all aspects of the work in ensuring that questions related to the accuracy or integrity of any part of the work are appropriately investigated and resolved. This study conducted in accordance with the Declaration of Helsinki (as revised in 2013). This study was approved by the Institutional Review Board of Aichi Cancer Center Hospital (ACC-2019-1-003). The requirement of informed consent was waived because of the retrospective nature of this study.

Open Access Statement: This is an Open Access article distributed in accordance with the Creative Commons Attribution-NonCommercial-NoDerivs 4.0 International License (CC BY-NC-ND 4.0), which permits the non- 
commercial replication and distribution of the article with the strict proviso that no changes or edits are made and the original work is properly cited (including links to both the formal publication through the relevant DOI and the license). See: https://creativecommons.org/licenses/by-nc-nd/4.0/.

\section{References}

1. Kuroda H, Mori S, Tanaka H, et al. Prognostic significance of combined radiologic imaging modalities for prognosis of clinical IA adenocarcinomas. Oncotarget 2018;9:10745-53.

2. Kuroda H, Nakada T, Oya Y, et al. Computed tomography and positron emission tomography-staged $\mathrm{cN} 0$ non-small cell lung cancer. Video-assist Thorac Surg 2020;5:14.

3. Conforti F, Pala L, Pagan E, et al. Effectiveness of intensive clinical and radiological follow-up in patients with surgically resected NSCLC. Analysis of 2661 patients from the prospective MAGRIT trial. Eur J Cancer 2020;125:94-103.

4. Ettinger DS, Wood DE, Aggarwal C, et al. NCCN Guidelines Insights: Non-Small Cell Lung Cancer, Version 1.2020. J Natl Compr Canc Netw 2019;17:1464-72.

5. Oya Y, Kuroda H, Nakada T, et al. Efficacy of Immune Checkpoint Inhibitor Monotherapy for Advanced NonSmall-Cell Lung Cancer with ALK Rearrangement. Int J Mol Sci 2020;21:2623.

6. Castello A, Rossi S, Toschi L, et al. Soluble PD-L1 in NSCLC Patients Treated with Checkpoint Inhibitors and Its Correlation with Metabolic Parameters. Cancers (Basel) 2020;12:1373.

7. Oya Y, Yoshida T, Kuroda H, et al. Predictive clinical parameters for the response of nivolumab in pretreated advanced non-small-cell lung cancer. Oncotarget 2017;8:103117-28.

8. Ferrucci PF, Ascierto PA, Pigozzo J, et al. Baseline neutrophils and derived neutrophil-to-lymphocyte ratio: prognostic relevance in metastatic melanoma patients receiving ipilimumab. Ann Oncol 2016;27:732-8.

9. Mori S, Usami N, Fukumoto K, et al. The Significance of the Prognostic Nutritional Index in Patients with Completely Resected Non-Small Cell Lung Cancer. PLoS One 2015;10:e136897.

10. Castello A, Toschi L, Rossi S, et al. The immunemetabolic-prognostic index and clinical outcomes in patients with non-small cell lung carcinoma under checkpoint inhibitors. J Cancer Res Clin Oncol
2020;146:1235-43.

11. Reck M, Rodríguez-Abreu D, Robinson AG, et al. Pembrolizumab versus Chemotherapy for PD-L1Positive Non-Small-Cell Lung Cancer. N Engl J Med 2016;375:1823-33.

12. Herbst RS, Baas P, Kim DW, et al. Pembrolizumab versus docetaxel for previously treated, PD-L1-positive, advanced non-small-cell lung cancer (KEYNOTE-010): a randomised controlled trial. Lancet 2016;387:1540-50.

13. Borghaei H, Paz-Ares L, Horn L, et al. Nivolumab versus Docetaxel in Advanced Nonsquamous Non-Small-Cell Lung Cancer. N Engl J Med 2015;373:1627-39.

14. Mok TSK, Wu YL, Kudaba I, et al. Pembrolizumab versus chemotherapy for previously untreated, PD-L1expressing, locally advanced or metastatic non-small-cell lung cancer (KEYNOTE-042): a randomised, open-label, controlled, phase 3 trial. Lancet 2019;393:1819-30.

15. Dafni U, Tsourti Z, Vervita K, et al. Immune checkpoint inhibitors, alone or in combination with chemotherapy, as first-line treatment for advanced non-small cell lung cancer. A systematic review and network meta-analysis. Lung Cancer 2019;134:127-40.

16. Wang C, Qiao W, Jiang Y, et al. The landscape of immune checkpoint inhibitor plus chemotherapy versus immunotherapy for advanced non-small-cell lung cancer: A systematic review and meta-analysis. J Cell Physiol 2020;235:4913-27.

17. Ichiki Y, Taira A, Chikaishi Y, et al. Prognostic factors of advanced or postoperative recurrent non-small cell lung cancer targeted with immune check point inhibitors. J Thorac Dis 2019;11:1117-23.

18. Shi Y, Au JS, Thongprasert S, et al. A prospective, molecular epidemiology study of EGFR mutations in Asian patients with advanced non-small-cell lung cancer of adenocarcinoma histology (PIONEER). J Thorac Oncol 2014;9:154-62.

19. Mizuno T, Yatabe $Y$, Kuroda H, et al. Impact of the oncogenic status on the mode of recurrence in resected non-small cell lung cancer. Jpn J Clin Oncol 2016;46:928-34.

20. Ni J, Guo T, Li Y, et al. Patterns and risks of postoperative recurrence in completely resected EGFR-mutant nonsmall cell lung cancer: prognostic significance of routine immunohistochemical markers. Transl Lung Cancer Res 2019;8:967-78.

21. Satouchi M, Nosaki K, Takahashi T, et al. First-line pembrolizumab vs chemotherapy in metastatic non-smallcell lung cancer: KEYNOTE-024 Japan subset. Cancer Sci 
2020;111:4480-9.

22. Mezquita L, Auclin E, Ferrara R, et al. Association of the Lung Immune Prognostic Index With Immune Checkpoint Inhibitor Outcomes in Patients With Advanced Non-Small Cell Lung Cancer. JAMA Oncol

Cite this article as: Kuroda $\mathrm{H}$, Takahashi $\mathrm{Y}$, Shirai S, Takahara H, Nakada T, Sakakura N, Matsushita H. Survival benefit of immune checkpoint inhibitor monotherapy in patients with non-small cell lung cancer recurrence after completely pulmonary resection. Ann Transl Med 2021;9(15):1225. doi: 10.21037/atm-21-1492
2018;4:351-7.

23. Khaki AR, Li A, Diamantopoulos LN, et al. Impact of performance status on treatment outcomes: A real-world study of advanced urothelial cancer treated with immune checkpoint inhibitors. Cancer 2020;126:1208-16. 


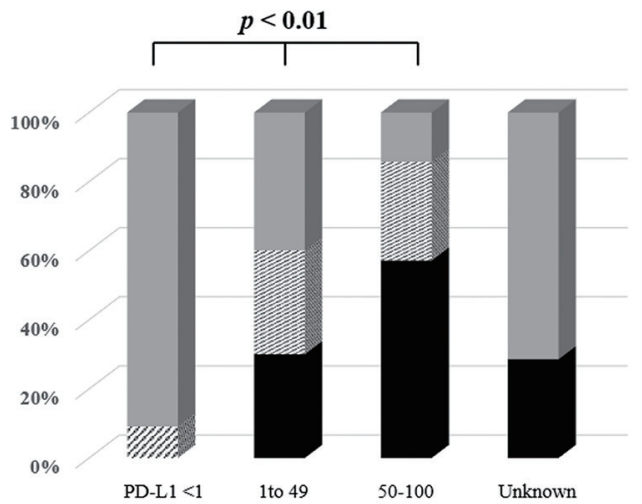

Figure S1 The proportion of the efficacious evaluation of immune checkpoint inhibitors Gray: Progressive Disease; Lattice: Stable Disease; and Black: Partial Response.

A

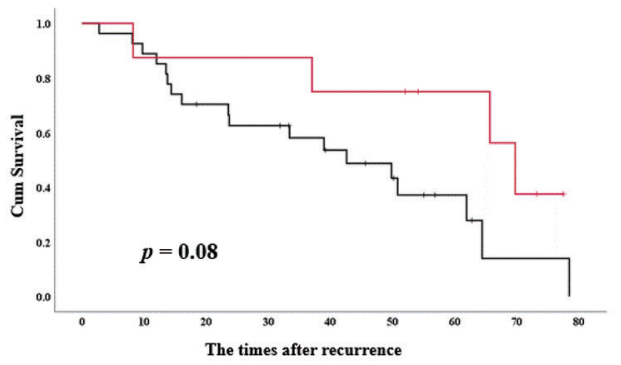

C

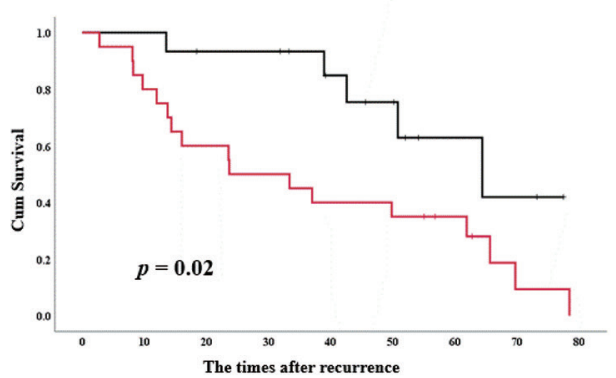

B

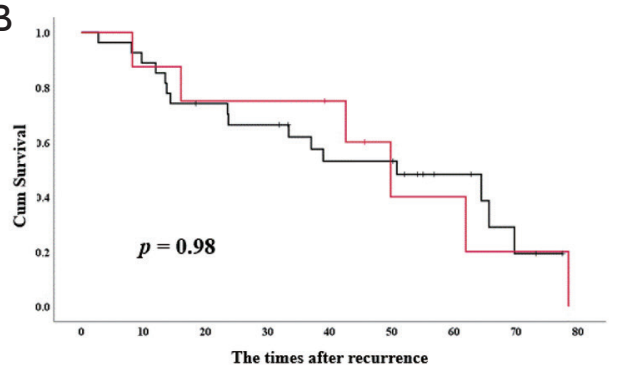

Figure S2 Kaplan-Meier curves. (A) The therapeutic outcomes after recurrence stratified by with (Red) and without (Black) EGFR or ALK. (B) those stratified by with (Red) and without (Black) radiation therapy before immune checkpoint inhibitors. EGFR: Epidermal growth factor receptor, and $A L K$, Anaplastic lymphoma kinase. (C) those stratified by DCR (Red) and no DCR (Black). The $\mathrm{P}<0.05$ means a significance. 